\title{
COEFICIENTES DE DIFUSÃO DE METAIS EM MATERIAIS NÃO CONVENCIONAIS (AGAROSE E ACETATO DE CELULOSE) USADOS NA TÉCNICA DE DIFUSÃO EM FILMES FINOS POR GRADIENTES DE CONCENTRAÇÃO
}

\author{
Camila Destro Colaço, Lauren Nozomi Marques Yabuki, Amanda Lodovico Alcântara e Amauri Antonio Menegário* \\ Centro de Estudos Ambientais, Universidade Estadual Paulista, Av. 24-A, 1515, 13506-900 Rio Claro - SP, Brasil
}

Recebido em 21/9/11; aceito em 15/2/12; publicado na web em 15/6/12

\begin{abstract}
DIFFUSION COEFFICIENTS OF METALS IN NON-CONVENTIONAL MATERIALS (AGAROSE AND CELLULOSE ACETATE) USED IN THE DIFFUSIVE GRADIENTS IN THIN FILMS TECHNIQUE. The DGT technique allows one to measure quantitatively free and labile metal species in aquatic systems. Nevertheless, for this approach, knowledge is required of the diffusion coefficients of the analytes in a diffusive layer. In this study, the diffusion coefficients of $\mathrm{Hg}$ (II), $\mathrm{As}$ (III), $\mathrm{Mn}$ (II), $\mathrm{Mg}$ (II), $\mathrm{Cu}$ (II), $\mathrm{Cd}$ (II) were determined in agarose gel and those of $\mathrm{Ba}(\mathrm{II}), \mathrm{Cd}(\mathrm{II}), \mathrm{Cu}(\mathrm{II}), \mathrm{Mg}(\mathrm{II}), \mathrm{Mn}(\mathrm{II})$ e $\mathrm{Zn}$ (II) in cellulose acetate membranes. These materials presented good performance and the reported results can be used as a data base for further DGT studies.
\end{abstract}

Keywords: DGT; agarose; cellulose acetate.

\section{INTRODUÇÃO}

A investigação da biodisponibilidade de metais na água é tão importante quanto a sua concentração ao considerar seu impacto em um ambiente. Informações sobre a reatividade, mobilidade e biodisponibilidade dos metais em águas naturais podem ser obtidas mediante estudos de especiação ou fracionamento químico. ${ }^{1,2}$

Dessa maneira, uma das maiores dificuldades encontradas na especiação de um elemento é o desenvolvimento de procedimentos analíticos que não alterem as condições reais do meio analisado. $\mathrm{O}$ estudo da reatividade do metal em um sistema aquático requer metodologia analítica adequada e procedimentos que permitam a análise in situ. ${ }^{3} \mathrm{O}$ uso de técnicas analíticas capazes de determinar diferentes espécies químicas com baixa manipulação amostral assume destaque com relação à compreensão da dinâmica dos metais no ambiente. ${ }^{4}$

A técnica de difusão em filmes finos por gradiente de concentração DGT baseia-se na $1^{\text {a }}$ Lei de Difusão de Fick que descreve, na forma de equação diferencial, a difusão de matéria em um meio no qual inicialmente não existe equilíbrio químico. Nesta situação estabelece-se um gradiente de concentração, produzindo um fluxo de partículas que tendem a homogeneizar a solução e uniformizar a concentração. ${ }^{5}$ Para a técnica DGT, as equações podem ser estabelecidas dessa forma:

O fluxo (F) dos íons que atravessam o gel difusivo para a resina ligante é expresso por:

$$
F=D \cdot\left(C_{b}-C^{\prime}\right) \cdot \Delta g^{-1}
$$

onde, D é o coeficiente de difusão do gel, $\mathrm{C}_{\mathrm{b}}$ é a concentração do metal lábil na solução externa, C' é a concentração do metal livre na resina e $\Delta \mathrm{g}$ é a espessura do gel difusivo.

A Equação 1 pode ser simplificada quando os íons metálicos estão em rápido equilíbrio com a resina apresentando uma forte ligação. Logo, a concentração C' será efetivamente zero:

$$
F=\left(D C_{b}\right) \cdot \Delta g^{-1}
$$

Pela definição de fluxo F = M. (At) ${ }^{-1}$, onde M é a massa do analito,

\footnotetext{
*e-mail: amenega@rc.unesp.br
}

A é a área da janela do dispositivo e t o tempo, a Equação 2 pode ser reescrita como:

$$
M=\left(D C_{b} t A\right) \cdot \Delta g^{-1}
$$

Determina-se a massa $\mathrm{M}$ do analito e calcula-se a concentração $\mathrm{C}_{\mathrm{b}}$ do metal na solução externa pela Equação 4:

$$
C_{b}=(M \Delta g) \cdot(D t A)^{-1}
$$

Convencionalmente, a camada difusiva se compõe de um filtro de ésteres mistos de celulose (filtro protetor) e um gel de poliacrilamida-agarose (agente difusivo propriamente dito). Como agente ligante emprega-se a resina Chelex-100 imobilizada em gel de poliacrilamida-agarose. ${ }^{6}$

A técnica DGT permite medir in situ quantitativamente a fração lábil dos metais solúveis (íons livres, todos os complexos inorgânicos e parte dos complexos orgânicos) em sistemas aquáticos, ${ }^{7}$ além de possibilitar a pré-concentração dos analitos, ${ }^{6}$ e sua determinação multielementar (quando associada às técnicas analíticas como a espectrometria de massa - ICP-MS - ou emissão ótica com plasma indutivamente acoplado - ICP OES).

Comparada à técnica ASV (voltametria de redissolução anódica), a DGT apresenta inúmeras vantagens: facilidade de uso em análises de rotina para monitoramento ambiental, realização de medidas in situ e rápida obtenção de resultados. Porém, o emprego do DGT requer o conhecimento prévio sobre a difusão do analito no hidrogel, a capacidade de retenção da resina Chelex e a limitação para sistemas com força iônica menor que $0,1 \mathrm{mmol} \mathrm{L}^{-1}$. A ASV proporciona como atrativo a possibilidade de se obter dados sobre as características de complexação das amostras. Contudo, suas adulterações na amostra podem ocorrer por se tratar de um procedimento moroso e ex situ. ${ }^{8}$

Em uma análise com DGT, os dispositivos são imersos in situ durante um período pré-estabelecido de tempo, acumulando-se assim os íons de interesse na fase ligante. ${ }^{9}$ Neste processo, a exatidão na determinação das concentrações dos metais lábeis requer informações prévias sobre a taxa de difusão das espécies metálicas. ${ }^{10}$

Alguns materiais alternativos foram investigados para serem usados como camada difusiva ${ }^{11-14}$ e como resina ligante..$^{15-17}$ 
Estudos preliminares propuseram o uso da agarose como agente difusivo para mercúrio, substituindo o agente convencional (poliacrilamida agarose) que reage com o analito. ${ }^{11}$ Além de mercúrio e alguns outros poucos elementos, há poucos dados sobre a difusão de metais na agarose.

A membrana de acetato de celulose vem sendo utilizada com frequência na DGT como camada protetora no dispositivo convencional. ${ }^{6}$ Apesar disso, não se tem informação sobre a difusão dos metais nesta membrana. Para os cálculos, assume-se, erroneamente, que a difusão dos metais nesta camada é a mesma do gel de poliacrilamida.

Neste trabalho, visando obter informações sobre o uso de materiais alternativos na DGT, foram determinados os coeficientes de difusão de $\mathrm{Hg}(\mathrm{II}), \mathrm{As}(\mathrm{III}), \mathrm{Mn}(\mathrm{II}), \mathrm{Mg}(\mathrm{II}), \mathrm{Cu}(\mathrm{II}), \mathrm{Cd}(\mathrm{II})$ no gel de agarose e de $\mathrm{Ba}(\mathrm{II}), \mathrm{Cd}(\mathrm{II}), \mathrm{Cu}(\mathrm{II}), \mathrm{Mg}(\mathrm{II}), \mathrm{Mn}$ (II) e $\mathrm{Zn}$ (II) na membrana de acetato de celulose, usando-se uma câmara de difusão.

\section{PARTE EXPERIMENTAL}

\section{Equipamentos e acessórios}

Foi utilizado um espectrômetro de emissão óptica com plasma acoplado indutivamente, marca GBC, modelo integra XL (Melbourne, Austrália) equipado com uma câmara de nebulização do tipo ciclônica (Glass Expansion, Melbourne, Austrália) e um nebulizador tipo V-Groove (Glass Expansion) ou com um nebulizador ultrassônico (CETAC, modelo U5000AT ${ }^{+}$. Para determinação de mercúrio foi utilizado um espectrômetro de fluorescência atômica com geração de vapor a frio (CV - AFS) (PS Analytical modelo Millenium Merlin, Inglaterra).

A câmara de difusão utilizada consiste basicamente de dois compartimentos de acrílico separados por um orifício e foi previamente descrita. ${ }^{6}$

\section{Reagentes e soluções}

Em todo o trabalho foram utilizados reagentes de grau analítico e água purificada em um sistema deionizador Milli-Q (Millipore, Bedford, MA, EUA) com resistividade de $18,2 \mathrm{~m} \Omega \mathrm{cm}$.

Foram utilizadas soluções-estoque contendo $1000 \mathrm{mg} \mathrm{L}^{-1} \mathrm{de} \mathrm{Hg}$, Mn, Cu, Cd, Ba e Zn (Specsol, São Paulo, Brasil) e Mg (J. T. Baker High Purity Standards, México). A solução estoque de As(III) foi preparada a partir de $\mathrm{As}_{2} \mathrm{O}_{3}$ (Merck, Darmstadt, Alemanha).

Para utilização na câmara de difusão, foram preparadas, a partir de diluições das soluções-estoque, soluções doadoras contendo:

- $\quad$ 1,7 $\mathrm{mg} \mathrm{L}^{-1} \mathrm{de} \mathrm{Cd}(\mathrm{II}),\left(\mathrm{pH}\right.$ próximo de 5, força iônica $0,1 \mathrm{~mol} \mathrm{~L}^{-1} \mathrm{e}$ temperatura de $\left.24,5^{\circ} \mathrm{C}\right)$, para o gel difusivo de acrilamida $(15 \%$, $\mathrm{m} / \mathrm{v})$ - agarose $(0,3 \%, \mathrm{~m} / \mathrm{v})$;

- $10 \mathrm{mg} \mathrm{L}^{-1}$ de $\mathrm{Hg}(\mathrm{II}), \mathrm{As}(\mathrm{III}), \mathrm{Mn}$ (II) e $\mathrm{Cu}(\mathrm{II})$ (pH próximo de 5 , força iônica $0,05 \mathrm{~mol} \mathrm{~L}^{-1}$ e temperatura de $24,5^{\circ} \mathrm{C}$ ), para o gel de agarose $3,0 \%(\mathrm{~m} / \mathrm{v})$;

- $5 \mathrm{mg} \mathrm{L}^{-1}$ de $\mathrm{Hg}(\mathrm{II}), \mathrm{Mn}$ (II), $\mathrm{Mg}$ (II), $\mathrm{Cd}(\mathrm{II})$ e $\mathrm{Cu}$ (II) (pH próximo de 5 , força iônica $0,05 \mathrm{~mol} \mathrm{~L}^{-1}$ e temperatura de $26^{\circ} \mathrm{C}$ ), para o gel agarose $1,5 \%(\mathrm{~m} / \mathrm{v})$;

- $10 \mathrm{mg} \mathrm{L}^{-1}$ de $\mathrm{Ba}$ (II) e $\mathrm{Mg}$ (II) (pH próximo de 5, força iônica 0,05 mol L ${ }^{-1}$ e temperatura de $27^{\circ} \mathrm{C}$ ), para a membrana de acetato de celulose;

- $5 \mathrm{mg} \mathrm{L}^{-1} \mathrm{de} \mathrm{Cd}(\mathrm{II}), \mathrm{Cu}$ (II), $\mathrm{Mn}$ (II) e $\mathrm{Zn}$ (II) (pH próximo de 5, força iônica $0,05 \mathrm{~mol} \mathrm{~L}^{-1}$ e temperatura de $23^{\circ} \mathrm{C}$ ), para a membrana de acetato de celulose.

Essas soluções tiveram os valores de $\mathrm{pH}$ ajustados com solução de $\mathrm{NaOH}$ (Merck) e $\mathrm{HCl}$ (J. T. Baker) e os valores das forças iônicas ajustados com solução padrão de $\mathrm{NaNO}_{3}$ (Merck). As soluções receptoras foram preparadas com parâmetros idênticos aos das soluções doadoras, porém sem a presença dos íons.

Foi preparada uma solução padrão $0,05 \mathrm{~mol} \mathrm{~L}^{-1}$ de $\mathrm{NaNO}_{3}$ a partir de dissolução de nitrato de sódio $1 \mathrm{~mol} \mathrm{~L}^{-1} \mathrm{NaNO}_{3}$ para armazenamento dos materiais testados (agarose e membrana de acetato de celulose).

Solução de $\mathrm{SnCl}_{2} 2 \%$ (m/v) (Caledon, Georgetown, Canadá) em $15 \%$ (v/v) de $\mathrm{HCl}$ foi utilizada no sistema de geração de vapor frio, para determinação de $\mathrm{Hg}(\mathrm{II})$.

As soluções das curvas analíticas usadas para análises por ICP OES e CV-AFS foram preparadas a partir da diluição de soluções estoque de $1000 \mathrm{mg} \mathrm{L}^{-1}$ de $\mathrm{Hg}, \mathrm{Mn}, \mathrm{Cu}, \mathrm{Cd}, \mathrm{Ba}, \mathrm{Zn}$ e $\mathrm{Mg}$ e solução padrão $1000 \mathrm{mg} \mathrm{L}^{-1}$ de As(III).

Para avaliar a câmara de difusão foi utilizado um gel difusivo de acrilamida $(15 \%, \mathrm{~m} / \mathrm{v})$ - agarose $(0,3 \%, \mathrm{~m} / \mathrm{v})$ obtido da DGT Research Ltd (Lancaster, Reino Unido)

Em todos os experimentos utilizou-se agarose NA (Pharmacia Biotech $\mathrm{AB}$, Georgetown, Canadá) para o preparo dos géis, que é de alta pureza e indicada para eletroforese de ácidos nucleicos. Especificações da agarose NA, temperatura de polimerização (gel $2 \%, \mathrm{~m} / \mathrm{v})=34-37{ }^{\circ} \mathrm{C}$; força do gel $(1,5 \%, \mathrm{~m} / \mathrm{v})=2400 \mathrm{~g} \mathrm{~cm}^{-2}$; eletroendo-osmose (EEO) $0,1 \pm 0,01 \%$; sulfato $<0,08-0,12 \%$ e umidade $<7 \%$. $^{18}$

Foram utilizados discos de acetato de celulose (porosidade 0,45

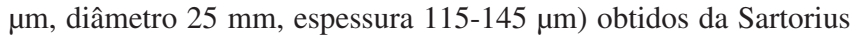
Stedim Biotech Ltd (Goettingen, Alemanha).

\section{Método}

Preparo da agarose

Foram preparados géis com composições de agarose de $1,5 \mathrm{e}$ $3,0 \%(\mathrm{~m} / \mathrm{v})$.

Sobre um aquecedor colocou-se um recipiente com aproximadamente $30 \mathrm{~mL}$ de água purificada. Uma vez atingida a temperatura de ebulição da água, adicionou-se a agarose ( 0,6 ou 1,2 g); a mistura resultante foi agitada até a total dissolução e, em seguida, o volume foi ajustado para $40 \mathrm{~mL}$. Após poucos minutos, a mistura foi prensada entre duas placas de vidro e deixada em repouso até seu resfriamento para formação do gel.

Depois de frio, o gel foi seccionado em forma de discos com 2,4 $\mathrm{cm}$ de diâmetro que, em seguida, foram imersos em solução 0,05 mol L-1 de $\mathrm{NaNO}_{3}$. Os géis apresentaram espessura média de 1,00 $\pm 0,05 \mathrm{~mm}$.

\section{Preparo do acetato de celulose}

Foram colocados 7 discos de acetato de celulose em solução de $10 \%$ (v/v) de $\mathrm{HNO}_{3}$ durante $24 \mathrm{~h}$. Em seguida, foram lavados com água purificada até $\mathrm{pH}$ próximo de 5 e imersos em uma solução 0,05 mol L ${ }^{-1}$ de $\mathrm{NaNO}_{3}$. Foram necessários 7 discos para resultar em uma espessura similar ao gel de poliacrilamida-agarose com o objetivo de minimizar o efeito da camada difusiva limite (diffusive boundary layer, DBL), que resulta em coeficientes de difusão subestimados. ${ }^{19}$

\section{Medida dos coeficientes de difusão com câmara de difusão}

A câmara de difusão usada, construída inteiramente de acrílico, possui dois compartimentos, ambos com capacidade de $70 \mathrm{~mL}$, separados por duas placas de acrílico, ambas com um orifício de $1,5 \mathrm{~cm}$ de diâmetro. Uma placa é fixa à câmara de difusão e a outra placa é móvel, para facilitar a inserção do agente difusivo.

$\mathrm{O}$ agente difusivo (gel de agarose ou membrana de acetato de celulose) foi colocado entre as duas placas, de modo que uma área de $1,77 \mathrm{~cm}^{2}$ ficasse exposta aos dois compartimentos. Em um dos compartimentos foi colocada a solução doadora e no outro a solução receptora, mantendo-se ambas as soluções em agitação constante. 
Alíquotas de igual volume foram retiradas de cada compartimento a cada 15 min, durante um período de no máximo 2 h. Na avaliação do desempenho da câmara para Cd(II), a retirada das alíquotas foi realizada em tempos pré-estabelecidos de 30, 60, 90, 100, 110, 120, 145 e $155 \mathrm{~min}$, pois o objetivo nesse experimento foi avaliar o desempenho da câmara de difusão e verificar o tempo de equilíbrio de difusão dos íons através do material difusivo.

Para todos os experimentos as alíquotas da solução receptora foram preservadas em $2 \%(\mathrm{v} / \mathrm{v}) \mathrm{HNO}_{3}$ para análise posterior.

Conhecida a temperatura da solução, a massa dos íons que passa pelo agente difusivo, a espessura, área de exposição dos agentes difusivos e, considerando que a concentração dos íons na solução de trabalho é constante, pode-se determinar o coeficiente de difusão (D) segundo a Equação 5:20

$$
D=(a \Delta g) \cdot\left(C_{A} S\right)^{-1}
$$

onde: $D$ é o coeficiente de difusão $\left(\mathrm{cm}^{2} \mathrm{~s}^{-1}\right) ; a$ é coeficiente angular da reta definida pela relação massa do íon no compartimento B por unidade de tempo ( $\mathrm{ng} \mathrm{s}^{-1}$ ); $\Delta g$ é a espessura do agente difusivo $(\mathrm{cm})$; $C_{\mathrm{A}}$ é a concentração do íon no compartimento $\mathrm{A}\left(\mathrm{mg} \mathrm{L}^{-1}\right)$ e $S$ é a área do orifício da câmara $\left(\mathrm{cm}^{2}\right)$.

A massa do íon difundida através do gel pode ser calculada pela Equação 6: ${ }^{20}$

$$
\mathrm{M}_{\mathrm{T}}=\mathrm{C}_{\mathrm{T}} \mathrm{V}_{\mathrm{T}}
$$

onde: $\mathrm{M}_{\mathrm{T}}$ - massa do íon difundida através do gel (ng); $\mathrm{C}_{\mathrm{T}}$ - concentração dos íons determinada no compartimento $\mathrm{B}\left(\mathrm{mg} \mathrm{L}^{-1}\right) ; \mathrm{V}_{\mathrm{T}}$ - volume da solução no compartimento B (mL).

\section{Correlação para medidas feitas em diferentes temperaturas}

A difusão é um processo dependente de três fatores, tamanho e forma do soluto, viscosidade do solvente e temperatura. A equação de Stokes - Einstein fornece uma relação aproximada entre coeficiente de difusão, viscosidade e temperatura. Essa relação está expressa na Equação 7:17,21

$$
\left(D_{1} \mu_{1}\right) \cdot\left(T_{1}\right)^{-1}=\left(D_{2} \mu_{2}\right) \cdot\left(T_{2}\right)^{-1}
$$

onde: $\mathrm{D}_{1}$ é o coeficiente de difusão do íon no gel determinado em $\mathrm{T}_{1}\left(\mathrm{~cm}^{2} \mathrm{~s}^{-1}\right) ; \mathrm{D}_{2}$ é o coeficiente de difusão do íon no gel determinado em $\mathrm{T}_{2}\left(\mathrm{~cm}^{2} \mathrm{~s}^{-1}\right) ; \mu_{1}$ é a viscosidade do solvente na temperatura $\mathrm{T}_{1}(\mathrm{~kg}$ $\left.(\mathrm{ms})^{-1}\right) ; \mu_{2}$ é a viscosidade do solvente na temperatura $\mathrm{T}_{2}\left(\mathrm{~kg}(\mathrm{~ms})^{-1}\right)$; $\mathrm{T}_{1}$ é a temperatura em que foi determinado o coeficiente $\left({ }^{\circ} \mathrm{C}\right) ; \mathrm{T}_{2}$ é a temperatura na qual se pretende encontrar o valor do coeficiente $\left({ }^{\circ} \mathrm{C}\right)$.

Para a agarose, visando facilitar a comparação dos resultados, todos os coeficientes de difusão determinados tiveram seus valores ajustados (usando a Equação 7) para uma temperatura de $25^{\circ} \mathrm{C}$. Para os cálculos, ao invés de viscosidade do gel de agarose foi adotada a viscosidade da água, pois os resultados para os coeficientes de difusão (D) no gel e na água são semelhantes. ${ }^{20}$

O mesmo procedimento não foi utilizado para a membrana de acetato de celulose, considerando-se o baixo nível de umidade nessa membrana.

A determinação de $\mathrm{As}$ (III), $\mathrm{Mn}$ (II), Cu(II), $\mathrm{Hg}$ (II), $\mathrm{Ba}$ (II), Cd(II), $\mathrm{Mg}(\mathrm{II})$ e $\mathrm{Zn}(\mathrm{II})$, para cada intervalo de tempo, foi realizada em espectrômetro de emissão ótica (ICP OES) equipado com nebulizador ultrassônico. As determinações de $\mathrm{Hg}$ (II) foram realizadas por espectrometria de fluorescência atômica com geração de vapor frio.

As determinações por ICP OES foram conduzidas utilizando as seguintes condições de operação: potência incidente $=1200 \mathrm{~W}$; gás plasma $=10 \mathrm{~L} \mathrm{~min}^{-1}$; gás auxiliar $=0,5 \mathrm{~L} \mathrm{~min}^{-1}$; gás de nebulização
$0,65 \mathrm{~L} \mathrm{~min}^{-1} ;$ modo de observação = radial; altura de observação = $10 \mathrm{~mm}$; temperatura de aquecimento (nebulizador ultrassônico) $=$ $142{ }^{\circ} \mathrm{C}$, temperatura de refrigeração (nebulizador ultrassônico) $=3^{\circ} \mathrm{C}$.

A determinação de $\mathrm{Hg}$ (II) com geração de vapor frio foi conduzida com $\mathrm{SnCl}_{2} 2 \%(\mathrm{~m} / \mathrm{v})$. Valor de linha de base $=5$; fator filtro $=8$; medição modo altura; modo de $=$ Razão; ganho $=10$; bomba peristáltica: bomba $1=100 \%$ (amostra), bomba $2=50 \%\left(\mathrm{SnCl}_{2}\right)$.

\section{RESULTADOS E DISCUSSÃO}

O desempenho da câmara de difusão foi previamente avaliado medindo-se o coeficiente de difusão de $\mathrm{Cd}(\mathrm{II})$ em um disco de gel difusivo convencional (acrilamida $(15 \%, \mathrm{~m} / \mathrm{v})-$ agarose $(0,3 \%, \mathrm{~m} / \mathrm{v})$ de $0,08 \mathrm{~cm}$ de espessura) e comparado-o com o valor tabelado.

Na Figura 1 são apresentadas as massas de cádmio encontradas no compartimento $\mathrm{B}$ por unidade de tempo. Observa-se na figura uma taxa de transferência constante de Cd(II) para o compartimento B para um intervalo de 30-120 min (correlação linear caracterizada por $\mathrm{R}^{2}$ $=0,9991$ ) e uma queda nesta taxa a partir de $130 \mathrm{~min}$ (devido a esse desempenho da câmara, nos experimentos seguintes realizados com a agarose e a membrana de acetato de celulose, o período de amostragem foi estabelecido até limite de $120 \mathrm{~min}$ ). Este comportamento é esperado segundo a Lei de Fick como consequiência da redução da concentração inicial do íon no compartimento A. ${ }^{6}$ Deste modo, para manter as condições de contorno da Equação 4, o coeficiente angular da faixa linear da curva $(0,200)$ foi utilizado para calcular o coeficiente de difusão.

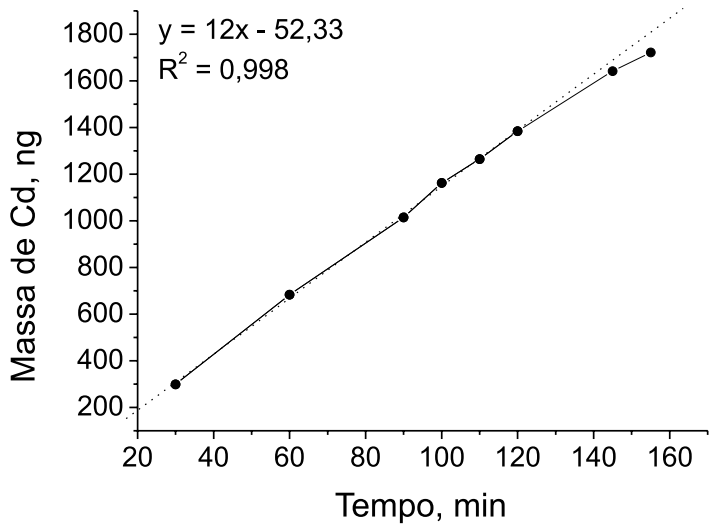

Figura 1. Difusão de cádmio no gel de acrilamida (15\%) - agarose (0,3\%). Dados obtidos usando uma câmara de difusão

Foi obtido um coeficiente de difusão $\left(24^{\circ} \mathrm{C}\right)$ de $5,39 \times 10^{-6} \mathrm{~cm}^{2}$ $\mathrm{s}^{-1}$, valor bastante próximo do valor esperado $\left(5,93 \times 10^{-6} \mathrm{~cm}^{2} \mathrm{~s}^{-1}\right),{ }^{20}$ evidenciando que a câmara desenvolvida pode ser utilizada satisfatoriamente para determinação de coeficientes de difusão de materiais alternativos e para determinação de coeficientes de difusão aparentes. Outra evidência do bom desempenho da câmara foram os resultados previamente relatados para determinação dos coeficientes de difusão de $\mathrm{Al}(\mathrm{III})$ e $\mathrm{Cu}(\mathrm{II}){ }^{22}$

\section{Coeficiente de difusão para gel de agarose $3,0 \%(\mathrm{~m} / \mathrm{v})$}

Os géis de composição 3,0 e 1,5\% (m/v) apresentaram consistência satisfatória para serem utilizados nos dispositivos. A resistência dos géis com composição 1,5 e 3,0\% (m/v) facilita o manuseio, especialmente quando preparados para uso em uma câmara de difusão. ${ }^{18}$ Visualmente, foi perceptível uma distinção entre os géis de agarose de 3,0 e $1,5 \%(\mathrm{~m} / \mathrm{v})$ devido à opacidade. O gel de composição $1,5 \%$ $(\mathrm{m} / \mathrm{v})$ apresentou-se mais translúcido do que o de $3,0 \%(\mathrm{~m} / \mathrm{v})$. 
A Tabela 1 apresenta as relações lineares das massas de As(III), $\mathrm{Hg}(\mathrm{II}), \mathrm{Cu}(\mathrm{II})$ e $\mathrm{Mn}$ (II) difundidas através do gel por unidade de tempo, nas alíquotas retiradas da solução receptora da câmara de difusão, depois de analisadas por ICP OES (equipado com nebulizador ultrassônico). Para todas as relações, observa-se claramente uma relação linear satisfatória $\left(\mathrm{R}^{2}\right.$ entre $\left.0,95-0,98\right)$ entre massa difundida e tempo de amostragem, o que indica que a primeira Lei de Fick foi seguida e, consequentemente, os dados obtidos podem ser utilizados para se determinar os coeficientes de difusão através dos analitos no gel de agarose $3,0 \%(\mathrm{~m} / \mathrm{v})$.

Tabela 1. Funções lineares obtidas da relação massa por tempo de $\mathrm{Hg}(\mathrm{II})$, $\mathrm{Cu}(\mathrm{II}), \mathrm{Mn}$ (II) e As(III) obtidas a partir de amostras retiradas da solução receptora em câmara de difusão usando agarose $3,0 \%(\mathrm{~m} / \mathrm{v})$

\begin{tabular}{cccc}
\hline \multirow{2}{*}{ Íon } & \multicolumn{3}{c}{ Coeficientes } \\
\cline { 2 - 4 } & Angular $\left(\mathrm{ng} \mathrm{s}^{-1}\right)$ & Linear $(\mathrm{ng})$ & $\mathrm{R}^{2}$ \\
\hline $\mathrm{Hg}(\mathrm{II})$ & 1,26 & 787,69 & 0,97 \\
$\mathrm{Cu}(\mathrm{II})$ & 0,73 & $-212,93$ & 0,95 \\
$\mathrm{Mn}(\mathrm{II})$ & 0,71 & 144,04 & 0,98 \\
$\mathrm{As}(\mathrm{III})$ & 0,84 & 691,47 & 0,97 \\
\hline
\end{tabular}

Os coeficientes de difusão dos íons As(III), $\mathrm{Hg}$ (II), $\mathrm{Cu}$ (II) e Mn(II) são apresentados na Tabela 2.

Tabela 2. Coeficiente de difusão (D) para $\mathrm{Hg}$ (II), $\mathrm{As}$ (III), $\mathrm{Mn}$ (II) e $\mathrm{Cu}$ (II) em agarose $3,0 \%(\mathrm{~m} / \mathrm{v}), \mathrm{pH}=5, \mathrm{I}=0,05 \mathrm{~mol} \mathrm{~L}^{-1} \mathrm{NaNO} 3$ e temperatura ajustada para $25^{\circ} \mathrm{C}$

\begin{tabular}{cccc}
\hline $\begin{array}{c}\text { Coeficiente de } \\
\text { difusão (D) }\end{array}$ & $\begin{array}{c}\text { Valores } \\
\text { encontrados } \\
\left(10^{-6} \mathrm{~cm}^{2} \mathrm{~s}^{-1}\right)\end{array}$ & $\begin{array}{c}\text { Valores em } \\
\text { água } \\
\left(10^{-6} \mathrm{~cm}^{2} \mathrm{~s}^{-1}\right)\end{array}$ & $\begin{array}{c}\text { Valores em } \\
\text { poliacrilamida } \\
\left(10^{-6} \mathrm{~cm}^{20,25} \mathrm{~s}^{-1}\right)\end{array}$ \\
\hline $\mathrm{Hg}(\mathrm{II})$ & $7,35 \pm 0,10$ & 10,74 & - \\
$\mathrm{As}(\mathrm{III})$ & $4,58 \pm 0,09$ & - & 6,80 \\
$\mathrm{Mn}(\mathrm{II})$ & $4,14 \pm 0,04$ & 6,88 & 9,84 \\
$\mathrm{Cu}(\mathrm{II})$ & $4,30 \pm 0,13$ & 7,33 & 10,49 \\
\hline
\end{tabular}

O coeficiente de difusão do $\mathrm{Hg}$ (II) determinado foi um pouco menor que o valor de referência para agarose $\left(8,97 \times 10^{-6} \mathrm{~cm}^{2} \mathrm{~s}^{-1}\right)^{11} \mathrm{e}$, também, para a difusão de mercúrio em água (solução do íon livre). ${ }^{24}$ Essa pequena diferença pode ser causada pelas composições dos géis que são diferentes (agarose 3,0\% (m/v) neste trabalho e agarose 1,5\% $(\mathrm{m} / \mathrm{v})$ no trabalho de referência) ou pela diferença da metodologia aplicada neste trabalho (câmara de difusão) e no trabalho de referência (imersão com dispositivos DGT).

$\mathrm{O}$ valor de coeficiente de difusão de As(III) em agarose 3,0\% $(\mathrm{m} / \mathrm{v})$ foi $\left(4,58 \times 10^{-6} \mathrm{~cm}^{2} \mathrm{~s}^{-1}\right)$ inferior ao valor relatado em gel de poliacrilamida $\left(6,80 \times 10^{-6} \mathrm{~cm}^{2} \mathrm{~s}^{-1}\right),{ }^{25}$ porém, a comparação de coeficientes de difusão em diferentes hidrogéis é mais complexa devido, por exemplo, a diferenças de porosidade e composição. ${ }^{9}$ Não se encontrou na literatura informações sobre coeficientes de difusão do As(III) em agarose. Os coeficientes de difusão determinados neste trabalho para $\mathrm{Mn}$ (II) e $\mathrm{Cu}$ (II) também foram inferiores aos valores relatados desses íons em água ${ }^{24}$ e poliacrilamida. ${ }^{20}$

Os íons $\mathrm{Ba}(\mathrm{II})$ e $\mathrm{Zn}$ (II) foram inicialmente avaliados para agarose $3,0 \%(\mathrm{~m} / \mathrm{v})$, porém os resultados obtidos não foram satisfatórios. Os valores encontrados indicaram uma possível contaminação da agarose para esses íons. Diante da dificuldade de descontaminação dos discos de géis de agarose, não se deu continuidade aos experimentos envolvendo a determinação dos coeficientes de difusão de $\mathrm{Ba}(\mathrm{II})$ e $\mathrm{Zn}(\mathrm{II})$.

\section{Coeficiente de difusão para gel de agarose $1,5 \%(\mathrm{~m} / \mathrm{v})$}

Na Tabela 3 são apresentas as relações das massas de $\mathrm{Mn}(\mathrm{II})$,
$\mathrm{Mg}(\mathrm{II}), \mathrm{Cd}(\mathrm{II}), \mathrm{Cu}$ (II) e $\mathrm{Hg}$ (II) difundidas através do gel de agarose $1,5 \%(\mathrm{~m} / \mathrm{v})$ encontradas nas alíquotas retiradas das soluções receptoras, em relação ao tempo. Semelhantemente aos resultados obtidos para o gel contendo agarose $3,0 \%(\mathrm{~m} / \mathrm{v})$, as relações massa por tempo de amostragem foram satisfatoriamente lineares.

Tabela 3. Funções lineares obtidas da relação massa por tempo de $\mathrm{Hg}(\mathrm{II})$, $\mathrm{Cu}(\mathrm{II}), \mathrm{Mn}(\mathrm{II}), \mathrm{Cd}(\mathrm{II})$ e $\mathrm{Mg}$ (II) ) obtidas a partir de amostras retiradas da solução receptora em câmara de difusão usando agarose $1,5 \%$ (m/v)

\begin{tabular}{cccc}
\hline \multirow{2}{*}{ Ín } & \multicolumn{3}{c}{ Coeficientes } \\
\cline { 2 - 4 } & Angular $\left(\mathrm{ng} \mathrm{s}^{-1}\right)$ & Linear $(\mathrm{ng})$ & $\mathrm{R}^{2}$ \\
\hline $\mathrm{Hg}(\mathrm{II})$ & 0,09 & 191,25 & 0,99 \\
$\mathrm{Cu}(\mathrm{II})$ & 0,34 & $-31,16$ & 0,98 \\
$\mathrm{Mn}(\mathrm{II})$ & 0,83 & $-1750,72$ & 0,99 \\
$\mathrm{Cd}(\mathrm{II})$ & 0,49 & $-878,96$ & 0,99 \\
$\mathrm{Mg}(\mathrm{II})$ & 0,75 & 314,25 & 0,99 \\
\hline
\end{tabular}

A Tabela 4 apresenta os coeficientes de difusão dos íons Mn(II), $\mathrm{Mg}(\mathrm{II}), \mathrm{Cd}(\mathrm{II}), \mathrm{Cu}(\mathrm{II})$ e $\mathrm{Hg}(\mathrm{II})$ no gel de agarose $1,5 \%$ (m/v).

Tabela 4. Coeficiente de difusão (D) para $\mathrm{Mn}(\mathrm{II}), \mathrm{Mg}(\mathrm{II}), \mathrm{Cd}(\mathrm{II}), \mathrm{Cu}(\mathrm{II}) \mathrm{e}$ $\mathrm{Hg}$ (II) em agarose $1,5 \%(\mathrm{~m} / \mathrm{v}), \mathrm{pH}=5, \mathrm{I}=0,05 \mathrm{~mol} \mathrm{~L}^{-1} \mathrm{NaNO}_{3}$ e ajustados para temperatura de $25{ }^{\circ} \mathrm{C}$

\begin{tabular}{cccc}
\hline $\begin{array}{c}\text { Coeficiente de } \\
\text { difusão (D) }\end{array}$ & $\begin{array}{c}\text { Valores } \\
\text { encontrados } \\
\left(10^{-6} \mathrm{~cm}^{2} \mathrm{~s}^{-1}\right)\end{array}$ & $\begin{array}{c}\text { Valores em } \\
\text { água } \\
\left(10^{-6} \mathrm{~cm}^{2} \mathrm{~s}^{-1}\right)\end{array}$ & $\begin{array}{c}\text { Valores em } \\
\text { poliacrilamida } \\
\left(10^{-6} \mathrm{~cm}^{2} \mathrm{~s}^{-1}\right)\end{array}$ \\
\hline $\operatorname{Mn}(\mathrm{II})$ & $8,85 \pm 0,03$ & 6,88 & 9,84 \\
$\operatorname{Mg}(\mathrm{II})$ & $7,98 \pm 0,06$ & 7,05 & - \\
$\mathrm{Cd}(\mathrm{II})$ & $5,22 \pm 0,01$ & 7,17 & 9,97 \\
$\mathrm{Cu}(\mathrm{II})$ & $4,82 \pm 0,01$ & 7,33 & 10,49 \\
$\mathrm{Hg}(\mathrm{II})$ & $7,21 \pm 0,01$ & 10,74 & - \\
\hline
\end{tabular}

Em agarose 1,5\% (m/v), os íons $\mathrm{Mn}(\mathrm{II})$ e $\mathrm{Mg}$ (II) apresentaram coeficientes de difusão (Tabela 4) próximos dos valores para água. ${ }^{24}$ Em comparação com o gel de poliacrilamida, o coeficiente de difusão do $\mathrm{Mn}(\mathrm{II})$ em agarose $1,5 \%$ (m/v) foi aproximadamente $10 \%$ menor. ${ }^{20}$ Não se encontrou na literatura informações sobre coeficiente de difusão de $\mathrm{Mg}$ no gel de poliacrilamida.

Para $\mathrm{Cu}(\mathrm{II})$ e $\mathrm{Cd}(\mathrm{II})$ os coeficientes encontrados foram aproximadamente 34 e $27 \%$, respectivamente, inferiores aos seus coeficientes em água e cerca de $50 \%$ menores dos que os valores indicados por outros autores em poliacrilamida. ${ }^{20}$ Valores dos coeficientes de Cd(II) e $\mathrm{Cu}(\mathrm{II})$ em agarose $1,5 \%$ (m/v) próximos aos valores encontrados na água foram relatados em trabalhos anteriores. ${ }^{20}$ Por outro lado, outros autore $^{23}$ relataram para Cd(II) coeficiente de difusão de 3,01 x 10-6 $\mathrm{cm}^{2} \mathrm{~s}^{-1} \mathrm{em}$ agarose $1,5 \%(\mathrm{~m} / \mathrm{v})$, valor inferior ao encontrado em água.

O coeficiente de difusão pode variar dependendo da composição da agarose. Isso pode ser observado nas Tabelas 2 e 4 para Mn(II), que apresentou um significativo aumento (maior que 50\%) quando a concentração de agarose foi reduzida de 3,0 para 1,5\% (m/v). Por outro lado, os íons $\mathrm{Hg}$ (II) e Cu(II) não indicaram variação entre diferentes composições de agarose, o que sugere que o íon $\mathrm{Mn}$ (II) possui uma maior interação com a agarose do que os íons $\mathrm{Hg}$ (II) e $\mathrm{Cu}$ (II).

Os valores dos coeficientes de difusão encontrados neste estudo para $\mathrm{Hg}(\mathrm{II})$ nos géis de agarose 3,0 e $1,5 \%(\mathrm{~m} / \mathrm{v}), 7,35 \pm 0,1 \times 10^{-6}$ $\mathrm{cm}^{2} \mathrm{~s}^{-1}$ e 7,21 $\pm 0,01 \times 10^{-6} \mathrm{~cm}^{2} \mathrm{~s}^{-1}$, respectivamente, foram semelhantes, mostrando que para esse íon a variação na composição de agarose não foi relevante. No entanto, o $\mathrm{Hg}$ (II) apresentou valor do coeficiente em agarose $1,5 \%(\mathrm{~m} / \mathrm{v})$ em média $30,5 \%$ menor que o relatado na literatura para agarose. É conhecido que o coeficiente de difusão pode variar de um tipo de agarose para outro. ${ }^{11}$ 


\section{Coeficiente de difusão em acetato de celulose}

Na Tabela 5 são apresentadas as relações das massas de Ba(II), $\mathrm{Cd}(\mathrm{II}), \mathrm{Cu}(\mathrm{II}), \mathrm{Mg}(\mathrm{II}), \mathrm{Mn}(\mathrm{II})$ e $\mathrm{Zn}(\mathrm{II})$ encontradas nas alíquotas retiradas das soluções receptoras, em relação ao tempo, para a membrana de acetato de celulose. Para todos os analitos observaram-se relações lineares.

Tabela 5. Funções lineares obtidas da relação massa por tempo de Ba(II), $\mathrm{Cd}(\mathrm{II}), \mathrm{Cu}(\mathrm{II}), \mathrm{Mg}(\mathrm{II}), \mathrm{Mn}(\mathrm{II})$ e $\mathrm{Zn}$ a partir de amostras retiradas da solução receptora em câmara de difusão usando membrana de acetato de celulose

\begin{tabular}{cccc}
\hline \multirow{2}{*}{ Ín } & \multicolumn{3}{c}{ Coeficientes } \\
\cline { 2 - 4 } & Angular $\left(\mathrm{ng} \mathrm{s}^{-1}\right)$ & Linear $(\mathrm{ng})$ & $\mathrm{R}^{2}$ \\
\hline $\mathrm{Ba}(\mathrm{II})$ & 0,33 & 12,85 & 0,98 \\
$\mathrm{Cd}(\mathrm{II})$ & 0,62 & $-570,10$ & 0,98 \\
$\mathrm{Cu}(\mathrm{II})$ & 0,86 & $-846,10$ & 0,94 \\
$\mathrm{Mg}(\mathrm{II})$ & 0,27 & 71,80 & 0,99 \\
$\mathrm{Mn}(\mathrm{II})$ & 0,63 & $-769,60$ & 0,99 \\
$\mathrm{Zn}(\mathrm{II})$ & 0,63 & $-159,10$ & 0,97 \\
\hline
\end{tabular}

Os valores de coeficientes de difusão encontrados para os íons $\mathrm{Ba}(\mathrm{II}), \mathrm{Cd}(\mathrm{II}), \mathrm{Cu}$ (II), $\mathrm{Mg}$ (II), $\mathrm{Mn}$ (II) e $\mathrm{Zn}$ (II) na membrana de acetato de celulose são indicados na Tabela 6 .

Tabela 6. Coeficiente de difusão (D) para $\mathrm{Ba}(\mathrm{II}), \mathrm{Cd}(\mathrm{II}), \mathrm{Cu}(\mathrm{II}), \mathrm{Mg}(\mathrm{II})$, $\mathrm{Mn}$ (II) e $\mathrm{Zn}$ (II) em membrana de acetato de celulose, $\mathrm{pH}=5$; I = 0,05 mol $\mathrm{L}^{-1} \mathrm{NaNO}_{3}$. Temperatura: $27{ }^{\circ} \mathrm{C}$ para $\mathrm{Ba}(\mathrm{II})$ e $\mathrm{Mg}(\mathrm{II}) ; 23{ }^{\circ} \mathrm{C}$ para $\mathrm{Cd}(\mathrm{II})$, $\mathrm{Cu}(\mathrm{II}), \mathrm{Mn}(\mathrm{II})$ e $\mathrm{Zn}(\mathrm{II})$

\begin{tabular}{cccc}
\hline $\begin{array}{c}\text { Coeficiente de } \\
\text { difusão (D) }\end{array}$ & $\begin{array}{c}\text { Valores encontra- Valores em água* } \\
\text { dos }\left(10^{-6} \mathrm{~cm}^{2} \mathrm{~s}^{-1}\right)\end{array}$ & $\begin{array}{c}\text { Valores em papel } \\
\left(10^{-6} \mathrm{~cm}^{2} \mathrm{~s}^{-1}\right)\end{array}$ & $\begin{array}{c}\text { cromatográfico** } \\
\left(10^{-6} \mathrm{~cm}^{2} \mathrm{~s}^{-1}\right)\end{array}$ \\
\hline $\mathrm{Ba}(\mathrm{II})$ & $1,47 \pm 0,09$ & 8,48 & - \\
$\mathrm{Cd}(\mathrm{II})$ & $3,01 \pm 0,09$ & 7,17 & 3,84 \\
$\mathrm{Cu}(\mathrm{II})$ & $3,50 \pm 0,20$ & 7,33 & 3,86 \\
$\mathrm{Mg}(\mathrm{II})$ & $1,39 \pm 0,05$ & 7,05 & - \\
$\mathrm{Mn}(\mathrm{II})$ & $3,37 \pm 0,08$ & 6,88 & 3,63 \\
$\mathrm{Zn}(\mathrm{II})$ & $3,40 \pm 0,10$ & 7,15 & 3,47 \\
\hline
\end{tabular}

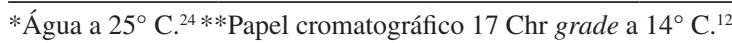

Todos os valores dos coeficientes de difusão encontrados foram inferiores aos valores relatados para água. ${ }^{24}$ Para os íons Ba(II) e $\mathrm{Mg}(\mathrm{II})$, a redução foi mais acentuada, chegando em média a $80 \%$. Para os demais íons, os coeficientes de difusão encontrados foram entre 50 a $60 \%$ inferiores aos coeficientes em água. ${ }^{24}$

Para os íons Cd(II), Cu(II), Mn(II) e Zn(II) os valores encontrados são bem próximos aos coeficientes de difusão desses íons no papel cromatográfico (17Chr grade). ${ }^{12}$ Os valores mais baixos para o papel cromatográfico, quando comparados com a poliacilamida e a água, provavelmente, não são devidos às restrições da mobilidade em sua estrutura, mas podem ser relacionados à quantidade de água e à baixa porosidade do papel cromatográfico (60\% de água) em relação à poliacrilamida ( $95 \%$ de água). ${ }^{12}$

Ainda não há relatos de determinação do coeficiente de difusão dos metais estudados em acetato de celulose.

\section{CONCLUSÕES}

Pela primeira vez são relatados coeficientes de difusão para Ba(II), $\mathrm{Cd}(\mathrm{II}), \mathrm{Cu}(\mathrm{II}), \mathrm{Mg}(\mathrm{II}), \mathrm{Mn}$ (II) e $\mathrm{Zn}(\mathrm{II})$ em acetato de celulose. Estes dados mostram-se extremamente importantes para o desenvolvimento e/ou uso da técnica DGT.

Os géis de agarose e a membrana de acetato de celulose utilizados permitiram uma difusão controlada dos íons $\mathrm{As}(\mathrm{III}), \mathrm{Ba}(\mathrm{II}), \mathrm{Cd}(\mathrm{II})$, $\mathrm{Cu}$ (II), $\mathrm{Hg}$ (II), $\mathrm{Mg}$ (II), $\mathrm{Mn}$ (II) e $\mathrm{Zn}$ (II). Os materiais testados tiveram um desempenho satisfatório e foram satisfatoriamente inertes em relação aos íons analisados, portanto, podem ser utilizados como agentes difusivos na técnica de difusão em filmes finos por gradiente de concentração (DGT) para determinação desses íons. A membrana de acetato de celulose é vantajosa devido à resistência à ruptura, fundamental para manipulação em laboratório e análises in situ, principal aplicação da técnica; para a agarose é necessário avaliar sua origem e composição, para estabelecer o coeficiente de difusão dos analitos.

\section{REFERÊNCIAS}

1. Balistrieri, L. S.; Blank, R. G.; Appl. Geochem. 2008, 23, 3355.

2. Wallner-Kersanach, W.; Andrade, C. F. F.; Zhang, H.; Milani, M. R.; Niencheski, L. F. H.; J. Braz. Chem. Soc. 2009, 20, 333.

3. Goveia, D.; Rosa, A. H.; Belli, I. C.; Lobo, F. A.; Fraceto, L. F.; Rove, J. A. F.; Romão, L. P. C.; Filho, N. L. D.; Anal. Bioanal. Chem. 2008, 390, 1173.

4. Tonietto, A. E.; Grassi, T. M.; Quim. Nova 2012, 35, 170.

5. Sissom, E. L.; Pitts, R. D. Em Elements of Transport Phenomena; Luiz, M. A., trad.; LTC: Rio de Janeiro, 1979, cap. 7.

6. Zhang, H.; Davison, W.; Anal. Chem. 1995, 67, 3391.

7. Vuillemim, M. H. T.; Gourlay, C.; Lorgraux, C.; Mouchel, J. M.; Buzier, R.; Gilbin, R.; Seidel, J. L.; Poulichet, F. E.; Sci. Total Environ. 2007, 375,244

8. Anjos, E. V.; Abate, G.; Grassi, T. M.; Quim. Nova 2010, 33, 1307.

9. Scally, S.; Davison, W.; Zhang, H.; Anal. Chim. Acta 2006, 558, 222.

10. Tonello, P. S.; Rosa, A. H.; Abreu Jr., C. H.; Menegário, A. A.; Anal. Chim. Acta 2007, 598, 162.

11. Docekalova, H.; Divis, P.; Talanta 2005, 65, 1174.

12. Larner, B. L.; Seen, A. J.; Anal. Chim. Acta 2005, 539, 349.

13. Martinez, R. A.; Gomez, M. M. G.; Greenwood, R.; Mills, G. A.; Vrana, B.; Corvillo, M. A. P.; Talanta 2009, 77, 1483.

14. Li, W.; Zhao, H.; Teasdale, P. R.; John, R.; Wang, F.; Anal. Chim. Acta 2005, 533, 193.

15. Li, W.; Zhao, H.; Teasdale, P. R.; John, R.; Zhang, S.; Anal. Chim. Acta 2002, 464, 331

16. Divis, P.; Szkandera, R.; Docekalova, H.; Central European Journal of Chemistry 2010, 8, 1102

17. Divis, P.; Szkandera,. R.; Brulik, L.; Docekalova, H.; Matús, P.; Bujdos, M.; Anal. Sci. 2009, 25, 575.

18. http://www.gelifesciences.com/aptrix/upp00919.nsf/Content/9B3A4D 61BE23C75FC1257628001CD11A/\$file/18101874.pdf, acessada em Abril 2012.

19. Garmo, O. A.; Royset, O.; Steinnes, E.; Flaten, T. P.; Anal. Chem. 2003, 75,3573 .

20. Zhang, H.; Davison, W.; Anal. Chim. Acta 1999, 398, 329.

21. http://books.google.com.br/books?id=TGRmfTrsPTQC\&pg=PA101\&h $\mathrm{l}=\mathrm{pt}-\mathrm{BR} \&$ source $=\mathrm{gbs} \_$toc_r $\& \mathrm{cad}=4 \# \mathrm{v}=$ onepage $\& \mathrm{q} \& \mathrm{f}=\mathrm{false}$, acessada em Abril 2012.

22. Tonello, P. S.; Rosa, A. H.; Abreu Jr, C. H.; Menegário, A. A.; Anal. Chim. Acta 2007, 598, 162.

23. Tecier, M. L.; Buffle, J.; Anal. Chem. 1996, 68, 3670.

24. Li, Y. H.; Gregory, S.; Geochim. Cosmochim. Acta 1974, 38, 704.

25. Panther, J. G.; Stillwell, K. P.; Powell, K. J.; Downard, A. J.; Anal. Chim. Acta 2008, 622, 133. 